\title{
Multi-Wavelength Observations of the Red Giant $R$ Doradus with the MAPPIT Interferometer
}

\author{
A.P. Jacob ${ }^{1}$, T.R. Bedding ${ }^{1}$, J.G. Robertson ${ }^{1}$, J.R. Barton, C.A. Haniff, \\ R.G. Marson \& M. Scholz \\ ${ }^{1}$ School of Physics, University of Sydney 2006, Australia
}

\begin{abstract}
We present visibility measurements of the nearby Mira-like star $\mathrm{R}$ Doradus taken over a wide range of wavelengths $(650-990 \mathrm{~nm})$. The observations were made using MAPPIT (Masked APerture-Plane Interference Telescope), an interferometer operating at the 3.9-m AngloAustralian Telescope. We used a slit to mask the telescope aperture and prism to disperse the interference pattern in wavelength. We observed in $\mathrm{R}$ Dor strong decreases in visibility within the $\mathrm{TiO}$ absorption bands. The results are in general agreement with theory but differ in detail, suggesting that further work is needed to refine the theoretical models.
\end{abstract}

Improvements in atmospheric models for $M$ giants requires measurements of stellar radii over a wide range of wavelengths. We present such observations for R Doradus (HR 1492; $V=5.4$; spectral type M8 IIIe), a nearby Mira-like star. We used MAPPIT (Masked APerture-Plane Interference Telescope), an interferometer operating at the 3.9-m Anglo-Australian Telescope. The telescope aperture was masked with a slit and a prism was used to disperse the interference pattern in wavelength.

We measured visibility over a wide range of wavelengths and compared our observations with the M-giant models by Bessell, Brett, Hofmann, Scholz and Wood (see Hofmann et al. 1998 and references within). We find agreement in the general features, with clear drops in visibility within the $\mathrm{TiO}$ absorption bands. However, although some models match the visibility observations in some regions, none match across the whole spectrum. The static $1 \mathrm{M}_{\odot}$ models, in particular, did not fit our observations well. The $1.2 \mathrm{M}_{\odot} \mathrm{M}$-series models (and $1 \mathrm{M}_{\odot} \mathrm{D}$ models), both of which pulsate in the fundamental mode, appear to be the best fits to our observations (see Fig. 1).

\section{References}

Hofmann, K. H., Scholz, M., \& Wood, P. R., 1998, A\&A, 339, 846.

Jacob, A. P., Bedding, T. R., Robertson, J. G., \& Scholz, M., 2000a, MNRAS, 312,733 .

Jacob, A. P., Bedding, T. R., Robertson, J. G., Barton, J. R., Haniff, C. A., Marson, R. G., \& Scholz, M., 2000b, In Lena, P. J., \& Quirrenbach, A., editors, Interferometry in Optical Astronomy, Proc. SPIE vol. 4406, page 723 (astro-ph/0005195). 


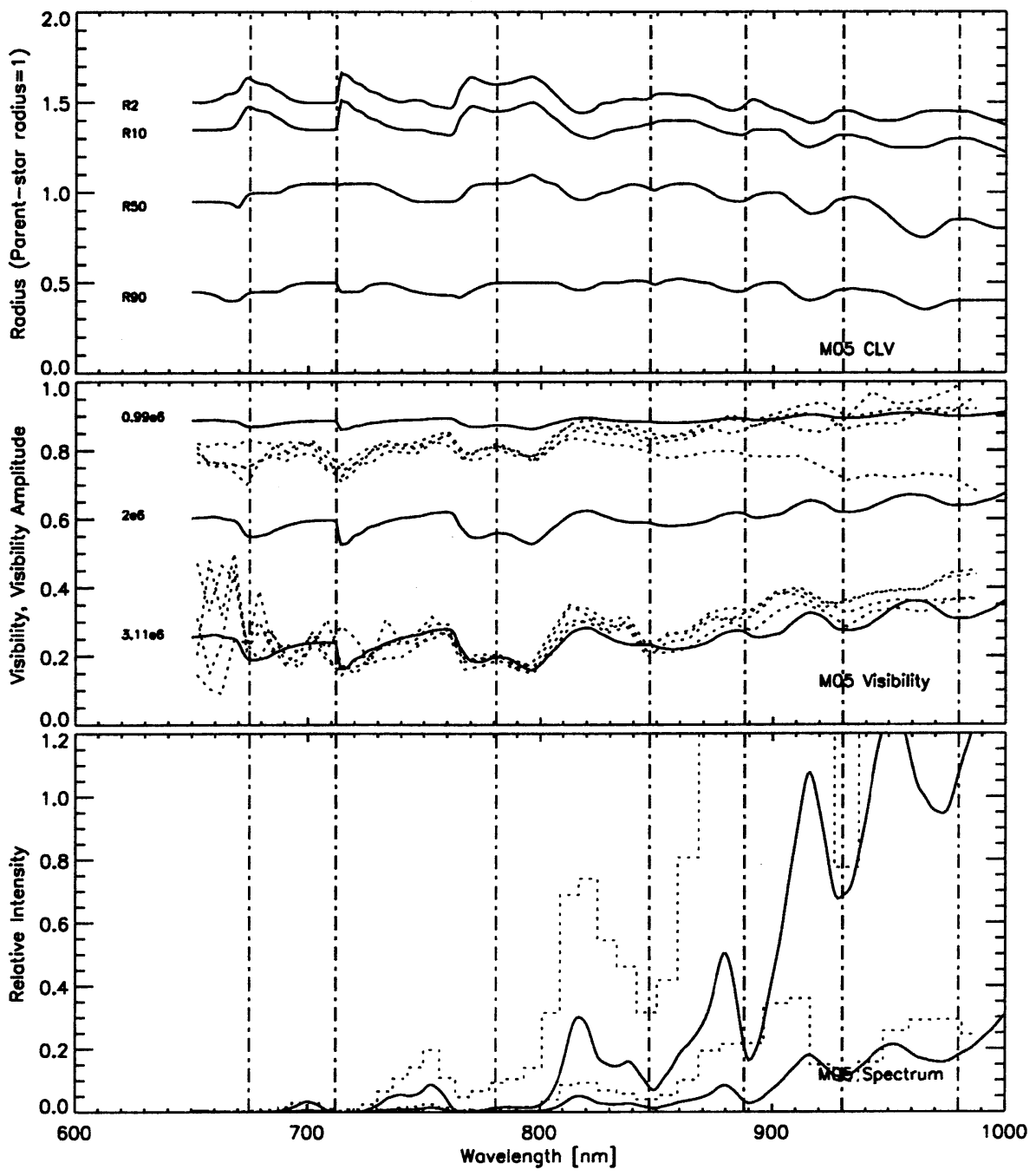

Figure 1. Comparison of observations of $\mathbf{R}$ Dor (dotted curves) with a $1.2 \mathrm{M}_{\odot}$ fundamental-mode model (M96400). The Rosseland angular diameter of the model was set to 53 mas. The top panel shows the model radius at 2, 10,50 and $90 \%$ of the central intensity. The middle panel shows model visibilities at three spatial frequencies, and the observed visibilities at two spatial frequencies. The bottom panel shows the flux spectrum for the model and observations, both plotted twice with vertical scales differing by a factor of 20 to accommodate the large range in intensities. To guide the eye, vertical dashed lines indicate the strongest absorption bands. The model calculations are described in full by Jacob et al. (2000a) and further details of the observations and discussion of the model fits are given by Jacob et al. (2000b). 